\title{
Optimization Method for Disturbance Compensation in Bilateral Control
}

\author{
Takuma Shimoichi* $^{*}$ Student Member, Seiichiro Katsura* ${ }^{*}$ Senior Member
}

(Manuscript received March 25, 2012, revised Nov. 19, 2012)

\begin{abstract}
Recently, real-world haptics which deals with real-world tactile sense has been actively researched. In real-world haptics, tactile information is extracted by a disturbance observer. In other words, tactile information is dealt with by an actuator as a disturbance force. Therefore, the disturbance of an actuator has to be minimized for the extraction of clearer tactile information and for the improvement of operationality. The identification and compensation of actuator's disturbance are necessary for improvement of operationality. However, in general, it is difficult to identify the disturbance because the disturbance has nonlinearity such as friction. In addition, the low accuracy of identification induces an overcompensation to the actuator.

In this paper, the method of improving operationality using optimization problem is proposed. A constraint optimization problem is constructed for the disturbance identification and compensation without overcompensation. The optimization problem is solved by a conjugate-direction method which involves an algorithm of nonlinear optimization. In addition, the proposed method can determine the parameter values of disturbance using only $10 \mathrm{~s}$ examination and automatic off-line processing. Therefore, considerable time and effort are not needed in the proposed method. The effectiveness of the proposed method is confirmed by experimental results.
\end{abstract}

Keywords: Real-World Haptics, Motion-Copying System, System Identification, Bilateral Control, Optimization, Haptic-Energy Conversion

\section{Introduction}

Recently, the technology of science has accomplished remarkable progress. The part of progress has been assumed by the craftsmanships of skilled craftsmen. Manufacturing technology of small and medium enterprises in Japan is representative example. There are some technologies which only some skilled craftsmen have such as metal molding, recognition of small difference in the tactile sense of object surface. However, recently, it becomes the problem that such technologies are being lost by the decrement of skilled craftsmen. Therefore, it is demanded that effective education and training for developing human resources that have practical techniques is established.

An imitative learning in the motion of skilled craftsmen is thought as the effective training method for the technique. An iteration of the imitative learning enables reproduction of the motion of skilled craftsmen. The imitative learning needs quantitative benchmarks of the motion such as trajectory and a level of force. The real-world haptics ${ }^{(1)}$ has been proposed for the imitation of trajectory in the human motion. In the real-world haptics, extraction, transmission, storage, analysis, redaction and reproduction of the human motion based on a bilateral control ${ }^{(2)-(5)}$ is researched. The bilateral control that is used for the real-world haptics is based on a disturbance observer (DOB) ${ }^{(6)(7)}$, reaction-force observer $(\text { RFOB })^{(8)-(10)}$ and modal transformation ${ }^{(11)}$. The robust accel-

\footnotetext{
* Department of System Design Engineering, Keio University 3-14-1, Hiyoshi, Kohoku-ku, Yokohama 223-8522, Japan
}

eration control of actuator is achieved by DOB. Force information which can not be obtained by the force sensor is able to be extracted by RFOB. In addition, the non-interacting control of position and force is achieved by the modal transformation. Therefore, position and force information of the human motion are extracted as the same time. Extracted information of the human motion is useful in the quantitative benchmarks of the motion.

The real-world haptics is achieved by using some kind of actuators. However, since the approach based on DOB extracts the haptic information as a disturbance force of actuators, pure haptic information can not be obtained when the disturbances such as a friction, a varied self-inertia torque, gravity force are included in the actuators. Therefore, the operationality of bilateral control is reduced. In order to deal with clear tactile information, an actuator which has a little mechanical friction should be used or, the disturbances have to be compensated by some sort of control. In the compensation of disturbances, the disturbance as haptic information must be separated with other disturbances. Therefore, modeling of other disturbance and its parameter identification are needed. Because of nonlinear characteristics in the disturbance of actuator, an identification is difficult. In addition, low accuracy of identification induces an overcompensation to the actuator. By the overcompensation, the haptic system with bilateral control is gone out of control.

In this paper, an identification method for disturbance parameters of actuator is proposed. The identified disturbance parameters using the proposed method does not induce the overcompensation to the actuator. The proposed method 


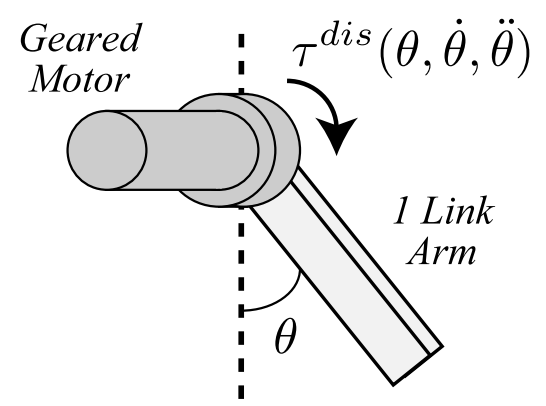

Fig. 1. System for disturbance identification

is based on a constraint optimization problem. The optimization problem is solved by a conjugate-direction method which is an algorithm of nonlinear optimization. Additionally, the proposed method can determine the parameter values of disturbance using a short examination and automatic off-line processing. Therefore, a lot of time and effort is not needed in the proposed method. The viability of the proposed method is confirmed by experimental results. The operationality of bilateral control is improved by the proposed method.

\section{Disturbance Model of Actuator}

In this part, the disturbance models of actuator are explained. The system for disturbance identification is shown in Fig. 1, where $\theta$ and $\tau$ denote the angle and torque, respectively. The superscript dis denotes disturbance. A varied selfinertia torque, gravity, and friction are focused on as the disturbances.

2.1 Varied Self-Inertia Torque A varied self-inertia torque $\tau_{s i}$ occurs when the actual and nominal actuator's inertia are different in the control. The torque $\tau_{s i}$ is represented as

$$
\begin{aligned}
\tau_{s i} & =\left(J-J_{n}\right) \ddot{\theta} \\
& =\Delta J \ddot{\theta} \ldots \ldots
\end{aligned}
$$

where $J$ denotes the inertia. In this section, $\Delta J$ is assumed to be constant. If $\Delta J$ is not constant, it is necessary to model $\Delta J$.

2.2 Gravity In the supposed system, the disturbance torque depending on the gravity is represented as

$$
\begin{aligned}
\tau_{g} & =M_{l} L_{G} g \sin \theta \\
& =M_{l g} g \sin \theta \cdots
\end{aligned}
$$

where $M_{l}, g, L_{G}$, and $M_{l g}$ denote the mass of link, acceleration of gravity, barycentric position of link and product of $M_{l}$, and $L_{G}$, respectively.

2.3 Friction Model A friction is generally assumed to consist of the Coulomb friction, viscous friction and Stribeck friction. The friction model which has continuity at zero speed and little number of parameters is constructed. The friction model is based on the time response of secondorder system $G(s)$ which is represent as

$$
G(s)=\frac{\omega_{n}^{2}}{\left(s+\omega_{n}\right)^{2}} .
$$

The response is described by the time function calculated by superposition of step, ramp and impulse inputs. To convert a

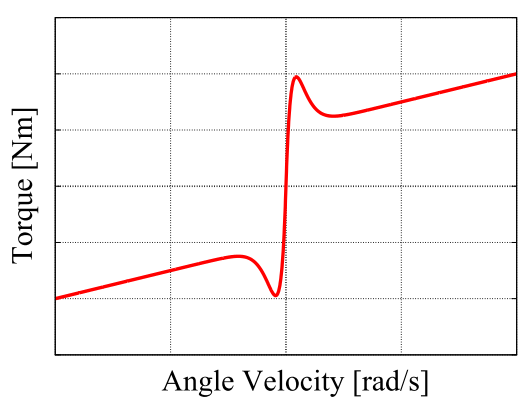

Fig. 2. Angle velocity-torque relation of friction model

time variable in the time function into velocity variable, the friction model is calculated as

$$
\begin{aligned}
& \tau_{s t}(\dot{\theta})=\tau_{c}\left(1-\left(1+\omega_{n} \dot{\theta}\right) \mathrm{e}^{-\omega_{n} \dot{\theta}}\right) \\
& \tau_{r m}(\dot{\theta})=\tau_{v}\left(1-\mathrm{e}^{-\omega_{n} \dot{\theta}}\right) \dot{\theta} \\
& \tau_{\text {im }}(\dot{\theta})=\tau_{s} \omega_{n}^{2} \dot{\theta} \mathrm{e}^{-\omega_{n} \dot{\theta}} \\
& \tau_{f r}(\dot{\theta})=\left(\tau_{s t}(|\dot{\theta}|)+\tau_{r m}(|\dot{\theta}|)+\tau_{\text {im }}(|\dot{\theta}|)\right) \operatorname{sgn}(\dot{\theta}) .
\end{aligned}
$$

The function based on the step response, ramp response, and impulse response represent the Coulomb friction, viscous friction, and Stribeck friction, respectively. The angle velocity-torque relation of (7) is shown in Fig. 2.

In addition, it is supposed that the parameters $\tau_{c}, \tau_{v}, \tau_{s}, \omega_{n}$ depend on a direction of velocity. Therefore, the parameters are represented as

$$
\begin{aligned}
\tau_{c} & = \begin{cases}\tau_{c}^{+} & (\dot{\theta}>0) \\
\tau_{c}^{-} & (\dot{\theta} \leq 0)\end{cases} \\
\tau_{v} & = \begin{cases}\tau_{v}^{+} & (\dot{\theta}>0) \\
\tau_{v}^{-} & (\dot{\theta} \leq 0)\end{cases} \\
\tau_{s} & = \begin{cases}\tau_{s}^{+} & (\dot{\theta}>0) \\
\tau_{s}^{-} & (\dot{\theta} \leq 0)\end{cases} \\
\omega_{n} & = \begin{cases}\omega_{n}^{+} & (\dot{\theta}>0) \\
\omega_{n}^{-} & (\dot{\theta} \leq 0) .\end{cases}
\end{aligned}
$$

These equations mean that $\tau_{c}, \tau_{v}, \tau_{s}$ and $\omega_{n}$ include two elements of real numbers.

In this paper, (1), (2) and (7) are used as the disturbance model. Therefore, the parameter vector of disturbance $\boldsymbol{Z}_{v}$ which needs to be determined is defined as

$$
Z_{v}=\left\{\Delta J, M_{l g}, \tau_{c}, \tau_{v}, \tau_{s}, \omega_{n}\right\}
$$

The parameter vector $\boldsymbol{Z}_{v}$ has 10 elements which are $\Delta J, M_{l g}$, $\tau_{c}^{+}, \tau_{c}^{-}, \tau_{v}^{+}, \tau_{v}^{-}, \tau_{s}^{+}, \tau_{s}^{-}, \omega_{n}^{+}$, and $\omega_{n}^{-}$.

\section{System Structure}

The structure of the proposed identification method is explained in this section. The method is based on the optimization problem and motion-copying system ${ }^{(12)}$. The procedures of the proposed method are shown as follows.

3.1 Procedure (i) The disturbance of actuator is extracted in this procedure. First, the system whose structure is same as the intended system for identification is prepared. These systems are implemented by a bilateral control. Instead of RFOB, DOB is used for the estimation of reactiontorque in the bilateral control system. The block diagram of 


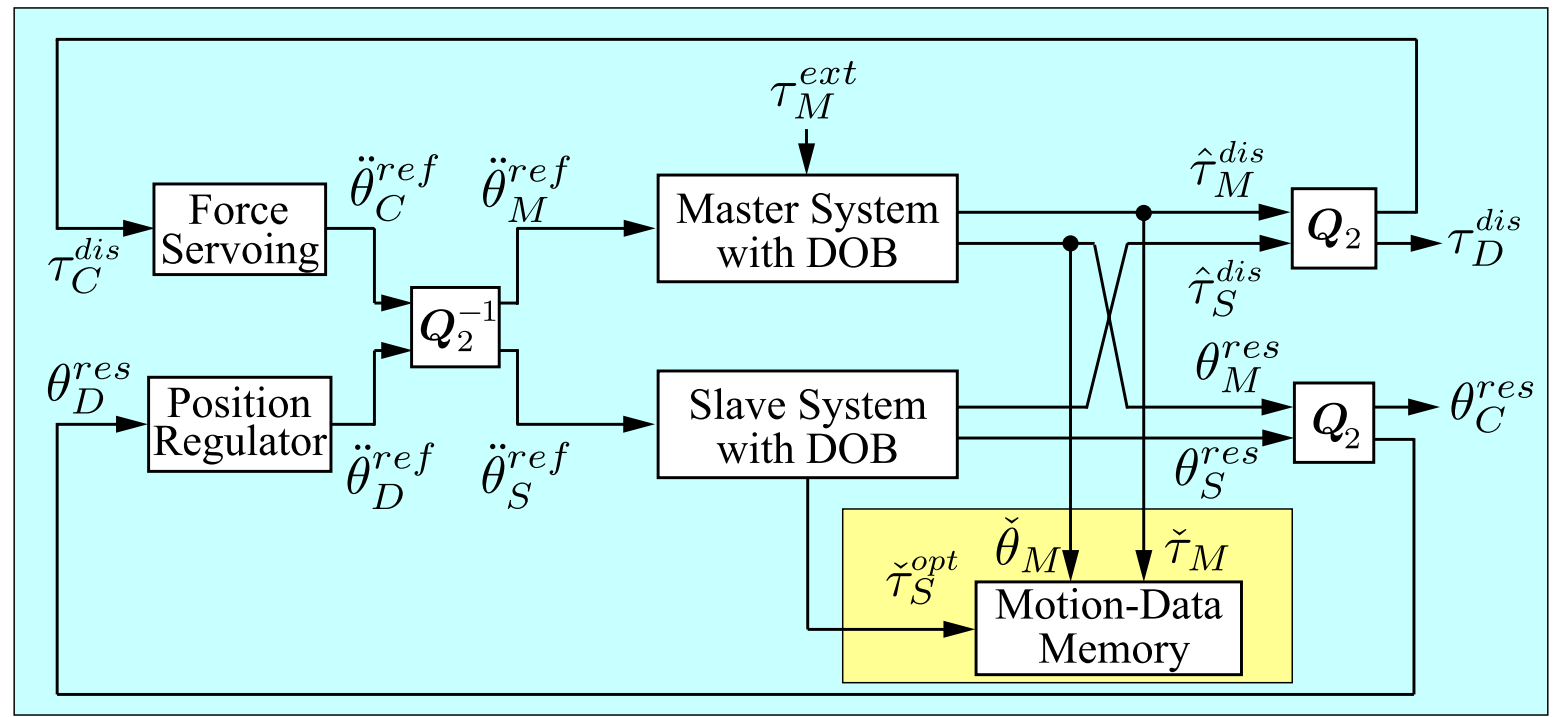

Fig. 3. Block diagram of procedure (i) of the proposed method

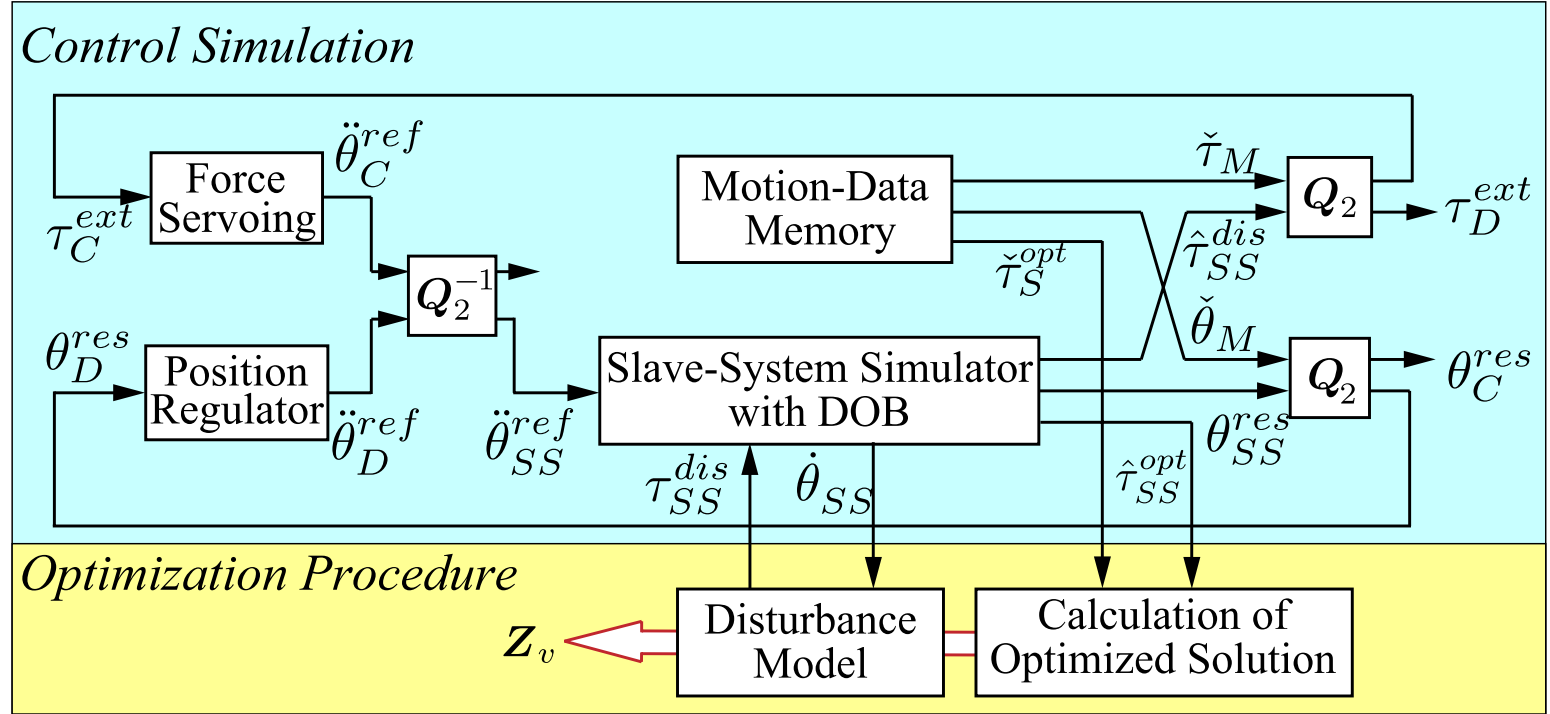

Fig. 4. Block diagram of procedure (ii) of the proposed method

this procedure is shown in Fig. 3. In Fig. 3, the subscripts $M, S, C$, and ${ }_{D}$ denote the master system, slave system, common mode, and differential mode, respectively. The superscripts ${ }^{r e f},{ }^{e x t},{ }^{r e s},{ }^{\wedge}$, and ${ }^{`}$ denote the reference value, external force, response value, estimate value, and saved value, respectively. Additionally, $\boldsymbol{Q}_{2}$ and $\boldsymbol{Q}_{2}^{-1}$ denote the secondorder quarry matrix and second-order inverse quarry matrix, respectively ${ }^{(13)} \cdot \boldsymbol{Q}_{2}$ is represented as

$$
\boldsymbol{Q}_{2}=\frac{1}{2}\left[\begin{array}{cc}
1 & 1 \\
1 & -1
\end{array}\right]
$$

The torque and angle responses of master system and slave system are coordinate-transformed into the common mode and differential mode by the quarry matrix, respectively

$$
\left[\begin{array}{c}
\tau_{C}^{d i s} \\
\tau_{D}^{d i s}
\end{array}\right]=\boldsymbol{Q}_{2}\left[\begin{array}{c}
\hat{\tau}_{M}^{d i s} \\
\hat{\tau}_{S}^{d i s}
\end{array}\right]
$$

$$
\left[\begin{array}{c}
\theta_{C}^{r e s} \\
\theta_{D}^{r e s}
\end{array}\right]=\boldsymbol{Q}_{2}\left[\begin{array}{c}
\theta_{M}^{r e s} \\
\theta_{S}^{r e s}
\end{array}\right]
$$

The control targets are represented as

$$
\begin{aligned}
\tau_{C}^{d i s} & =0 \\
\theta_{D}^{r e s} & =0 .
\end{aligned}
$$

To satisfy (16) and (17), the acceleration reference values are calculated by the force servoing and position regulator. The acceleration reference values of common mode $\ddot{\theta}_{C}^{r e f}$ and differential mode $\ddot{\theta}_{D}^{r e f}$ are represented as (18) and (19), respectively

$$
\begin{aligned}
& \ddot{\theta}_{C}^{r e f}=-K_{f} \tau_{C}^{d i s} \ldots \ldots \ldots \\
& \ddot{\theta}_{D}^{r e f}=-\left(K_{p}+K_{d} s\right) \theta_{D}^{d i s}
\end{aligned}
$$

where $s, K_{f}, K_{p}$, and $K_{d}$ denote the Laplace operator, force gain, position gain, and velocity gain, respectively. 


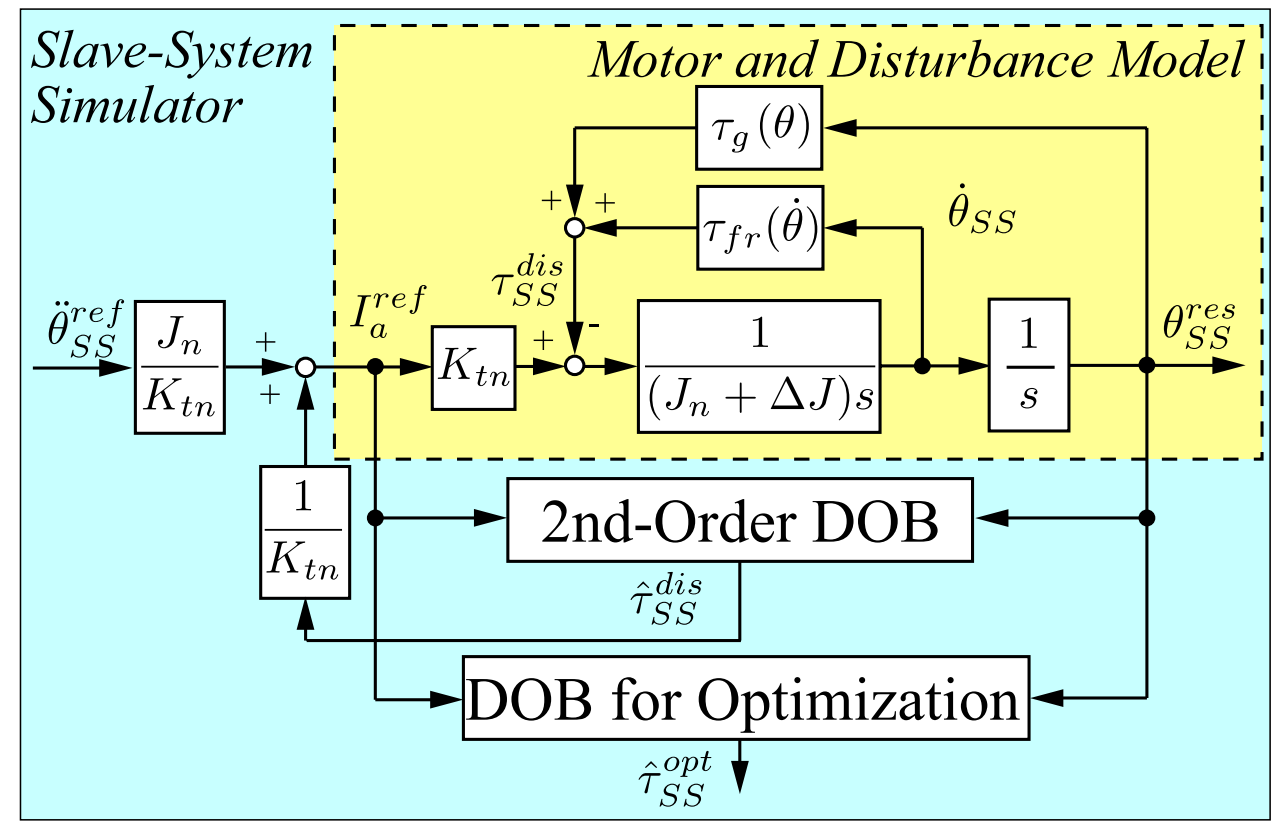

Fig. 5. Block diagram of slave-system simulator for the proposed method

$\tau_{S}^{o p t}$ denotes the disturbance torque that is estimated by DOB which is not used in the control. The cutoff frequency of DOB for $\tau_{S}^{o p t}$ is designed depending on the characteristics of disturbance. Like the motion-copying system, the torque and angle responses of the master system and $\tau_{S}^{o p t}$ are saved by the bilateral control under free motion. In this free motion, required motion patterns differ depending on the disturbance model. If the disturbance model that is explained in section 2 is adopted, the motion patterns should include the low velocity motion to obtain the characteristic of friction in low velocity.

3.2 Procedure (ii) The simulations of bilateral control are performed. The block diagram of procedure (ii) is shown in Fig. 4. In Fig. 4, the subscript ${ }_{S S}$ denotes the slavesystem simulator. The block diagram of slave-system simulator is shown in Fig. 5.

The performance index $I$ for the calculation of optimized solution is considered

$$
I\left(\boldsymbol{Z}_{v}\right)=\sum_{t=0}^{T_{a l l}}\left(\check{\tau}_{S}^{o p t}-\hat{\tau}_{S S}^{o p t}\left(\boldsymbol{Z}_{v}\right)\right)^{2}
$$

However, if the waveform of $\hat{\tau}_{S S}^{\text {opt }}\left(\boldsymbol{Z}_{v}\right)$ exceeds that of $\check{\tau}_{S}^{\text {opt }}$, the overcompensation occurs in the disturbance compensation. Therefore, the performance index $I_{s}\left(\boldsymbol{Z}_{v}\right)$ with scaling factor $\alpha$ is used. $I_{s}\left(\boldsymbol{Z}_{v}\right)$ is represented as

$$
I_{S}\left(\boldsymbol{Z}_{v}\right)=\sum_{t=0}^{T_{\text {all }}}\left(\alpha \check{\tau}_{S}^{\text {opt }}-\hat{\tau}_{S S}^{o p t}\left(\boldsymbol{Z}_{v}\right)\right)^{2} \quad(\alpha<1.0) . \cdots
$$

In this paper, $\alpha$ is set to 0.99 . Additionally, the constraint condition has to be considered for the optimization problem. In order to calculate the constraint optimization problem, a penalty index is introduced to the performance index. The penalty index $P$ is represented as

$$
P\left(\boldsymbol{Z}_{v}\right)= \begin{cases}\sum_{t=0}^{T_{a l l}}\left(\left|\check{\tau}_{S}^{\text {opt }}\right|-\left|\hat{\tau}_{S S}^{\text {opt }}\left(\boldsymbol{Z}_{v}\right)\right|\right)^{2} & (A \leq 0) \\ 0 & (A>0)\end{cases}
$$

where $A$ denotes $\left|\check{\tau}_{S}^{\text {opt }}\right|-\left|\hat{\tau}_{S S}^{\text {opt }}\left(\boldsymbol{Z}_{v}\right)\right|$. Thus, the performance in$\operatorname{dex} I_{p}$ which is used in the proposed method is represented as

$$
I_{p}\left(\boldsymbol{Z}_{v}\right)=I_{s}\left(\boldsymbol{Z}_{v}\right)+W P\left(\boldsymbol{Z}_{v}\right)
$$

where $W$ denotes weight coefficient. In this paper, $W$ is set to 10 .

A minimization algorithm of some sort is necessary to solve the constraint optimization problem. Because the performance index has many parameters, the optimization problem is too complex. Therefore, an algorithm not to use a derivation of a performance index with respect to a parameter vector is recommended. In this paper, the conjugate-direction method $^{(14)}$ is used to solve the constraint optimization problem. The simulation is iterated by the conjugate-direction method until the optimized solution is solved.

Generally, disturbances of actuator such as inertia, gravity, Coulomb friction, viscous friction and so on are separately identified by some sort of examinations. In the proposed method, all disturbance of actuator can be identified at a time by one examination. Therefore, with consideration for all disturbance, disturbance compensation without overcompensation can be achieved.

\section{Experiments}

In this section, the viability of the proposed method is verified by the experimental results. There are two kinds of experiments. One is the experiment for identification of actuator's disturbance. The other is the experiment of disturbance compensation. 


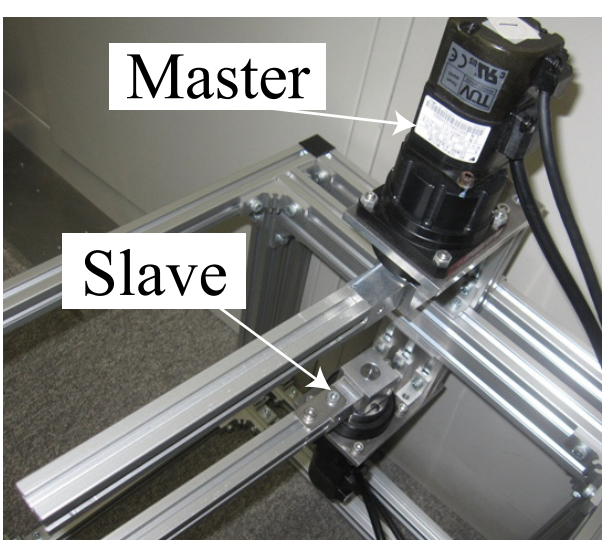

Fig. 6. Experimental system

Table 1. Experimental parameters for procedure (i) of the proposed method

\begin{tabular}{|c|c|c|}
\hline \hline Parameters & Descriptions & Values \\
\hline \hline$T_{s}$ & Sampling time & $100 \mu \mathrm{s}$ \\
$K_{t n}$ & Nominal force coefficient & $5.181 \mathrm{Nm} / \mathrm{A}$ \\
$J_{n}$ & Nominal inertia & $0.018 \mathrm{kgm}^{2}$ \\
$K_{f}$ & Force gain & 10 \\
$K_{p}$ & Position gain & 4225 \\
$K_{v}$ & Velocity gain & 130 \\
$g_{p d}$ & Cutoff frequency of & $10000 \mathrm{rad} / \mathrm{s}$ \\
& pseudo-deviation & \\
$g_{d i s}$ & Cutoff frequency of DOB & $300 \mathrm{rad} / \mathrm{s}$ \\
$g_{o p t}$ & Cutoff frequency of DOB & $100 \mathrm{rad} / \mathrm{s}$ \\
& for optimization & \\
\hline \hline
\end{tabular}

4.1 Experiments of Identification In this part, identification of actuator's disturbance is performed by the proposed method. The experimental system is shown in Fig. 6. The system consists of geared motor whose gear ratio is $1: 33$ and encoder whose resolution is 2048 pulse/rev. Control program is implemented in Real-Time Application Interface (RTAI) 3.7. The experimental parameters of procedure (i) are shown in Table 1 . The values of each gain and cutoff frequency are set as maximum value in the experimental system. The sampling time of slave-system simulator and its disturbance is 100 times faster than control cycle.

The results of the performance index v.s. number of iteration in the optimization are shown in Fig. 7 and Fig. 8. In addition, the behaviors of parameter vector $\boldsymbol{Z}_{v}$ in the act of optimization are shown in Fig. 9. The initial values of $\boldsymbol{Z}_{v}$ are set to 0 . However, if $\omega_{n}^{+}$and $\omega_{n}^{-}$are equal to 0 , the output of friction model becomes 0 even if the other parameter values are changed. Therefore, only $\omega_{n}^{+}$and $\omega_{n}^{-}$are set to 10 .

The identified parameters using proposed method are shown in Table 2. The identified parameters are obtained by the optimization. Therefore, these are not necessarily correspond to the true values. However, the performance of bilateral control is improved by the disturbance compensation using these values. The responses of $\check{\tau}_{S}$ and $\hat{\tau}_{S S}^{\text {ext }}$ with the optimized parameter values are shown in Fig. 10. The responses are found to be identical by these figures. Fig. 11 shows the angle velocity-torque relation of $\check{\tau}_{S}$ and $\hat{\tau}_{S S}^{\text {ext }}$ with the optimized parameter. The angle velocity-torque relation of $\check{\tau}_{S}$ is different from that of the friction model. This would be

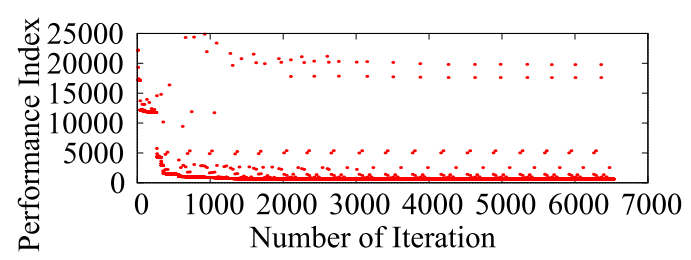

Fig. 7. Results of performance index v.s. number of iteration in the optimization

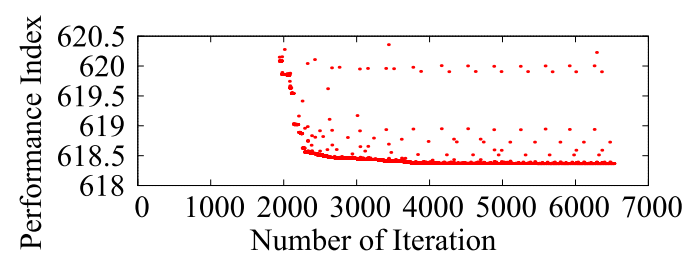

Fig. 8. Extended figure of Fig. 7
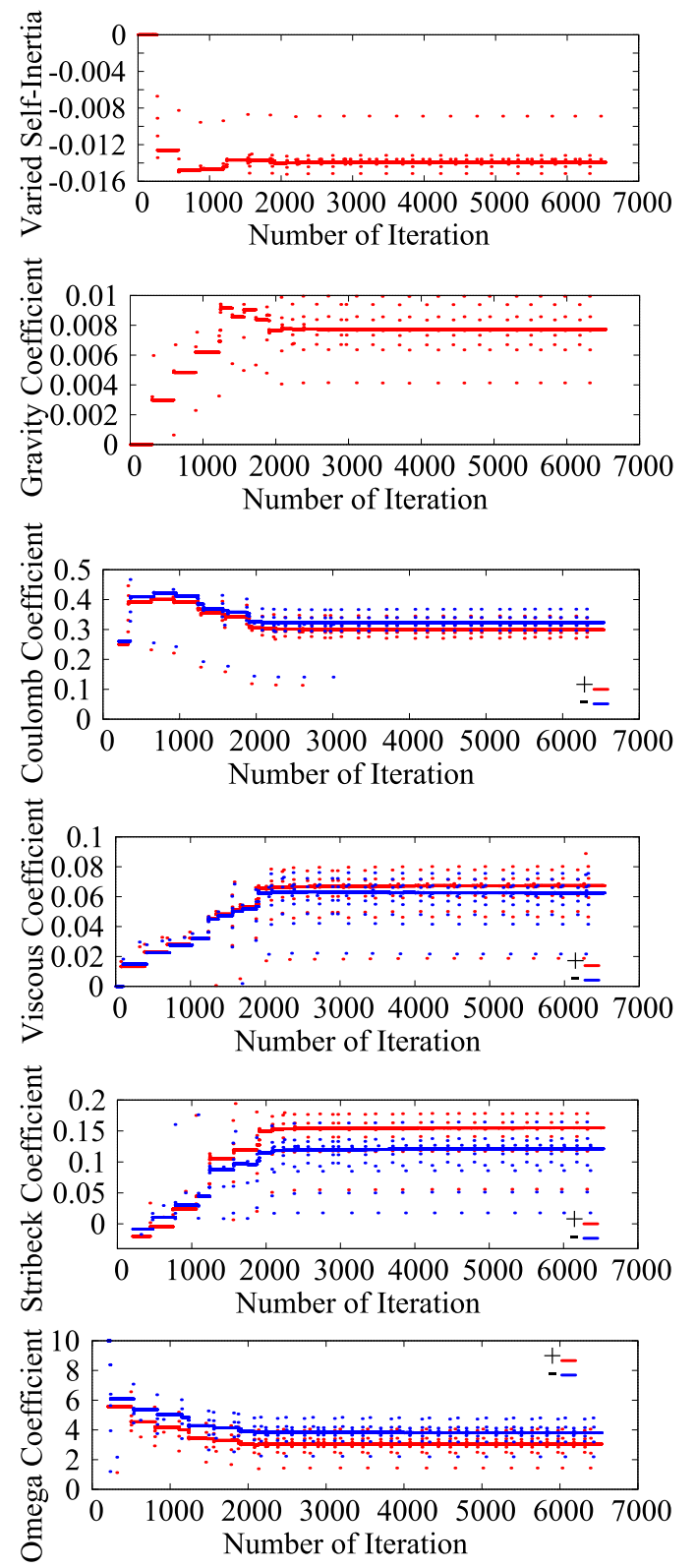

Fig. 9. Behaviors of $\boldsymbol{Z}_{v}$ in the act of optimization 
Table 2. Identified disturbance parameters

\begin{tabular}{|c|c|c|}
\hline \hline Parameters & Descriptions & Values \\
\hline \hline$\Delta J$ & Varied self-inertia & $-0.0139 \mathrm{kgm}^{2}$ \\
$M_{l g}$ & Gravity coefficient & $0.00770 \mathrm{kgm}$ \\
$\tau_{c}^{+}$ & Coulomb coefficient (+) & $0.299 \mathrm{Nm}$ \\
$\tau_{c}^{-}$ & Coulomb coefficient (-) & $0.324 \mathrm{Nm}$ \\
$\tau_{v}^{+}$ & Viscous coefficient (+) & $0.0673 \mathrm{Ns}$ \\
$\tau_{v}^{-}$ & Viscous coefficient (-) & $0.0626 \mathrm{Ns}$ \\
$\tau_{s}^{+}$ & Stribeck coefficient (+) & $0.156 \mathrm{Nm}^{2} / \mathrm{s}$ \\
$\tau_{s}^{-}$ & Stribeck coefficient (-) & $0.121 \mathrm{Nm}^{2} / \mathrm{s}$ \\
$\omega_{n}^{+}$ & Omega coefficient (+) & $3.04 \mathrm{~s} / \mathrm{m}$ \\
$\omega_{n}^{-}$ & Omega coefficient (-) & $3.81 \mathrm{~s} / \mathrm{m}$ \\
$I\left(\boldsymbol{Z}_{v}\right)$ & Performance index & 618 \\
\hline \hline
\end{tabular}
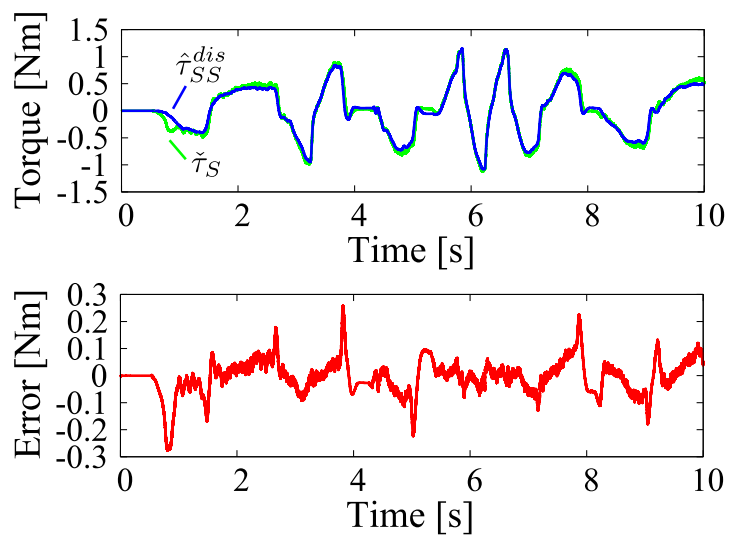

Fig. 10. Torque and error responses of $\check{\tau}_{S}$ and $\hat{\tau}_{S S}^{\text {ext }}$

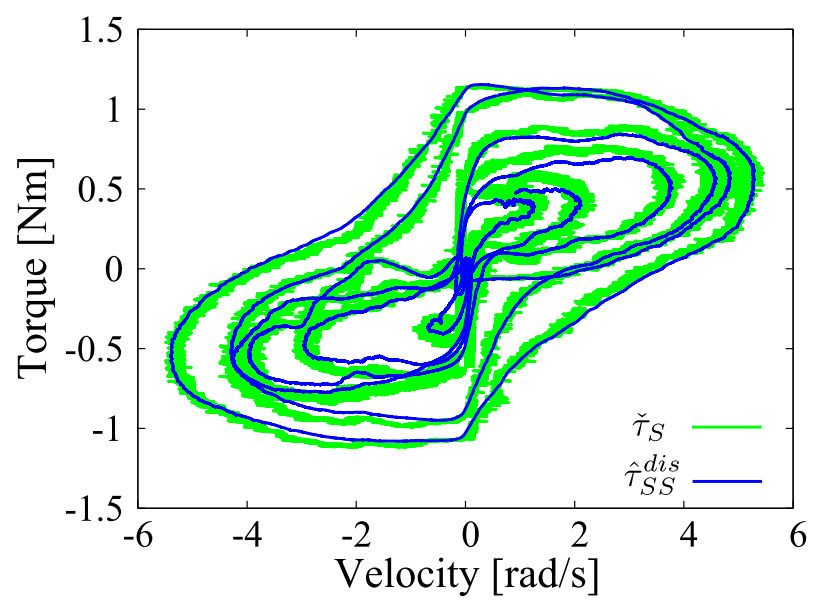

Fig. 11. Angle velocity-torque relation of $\check{\tau}_{S}$ and $\hat{\tau}_{S S}^{\text {ext }}$

caused by the varied self-inertia torque and gravity force. It is imagined easily by a comparison between the angle velocitytorque relation of $\check{\tau}_{S}$ and that of $\hat{\tau}_{S S}^{e x t}$ with the consideration of the varied self-inertia torque and gravity force.

In this optimization problem, it is thought that local minimums exist. The parameters determined by the proposed method don't necessarily correspond to the true optimized solution. In the conventional research ${ }^{(15)}$, the friction parameters which are near to the true optimized solution are identified by the optimization of simulated annealing. However, about 300,000 times of iteration of simulation is necessary for identification of 5 parameters. The number of iteration of the conventional research ${ }^{(15)}$ are shown in Fig. 15, where $F_{f}^{c}$,
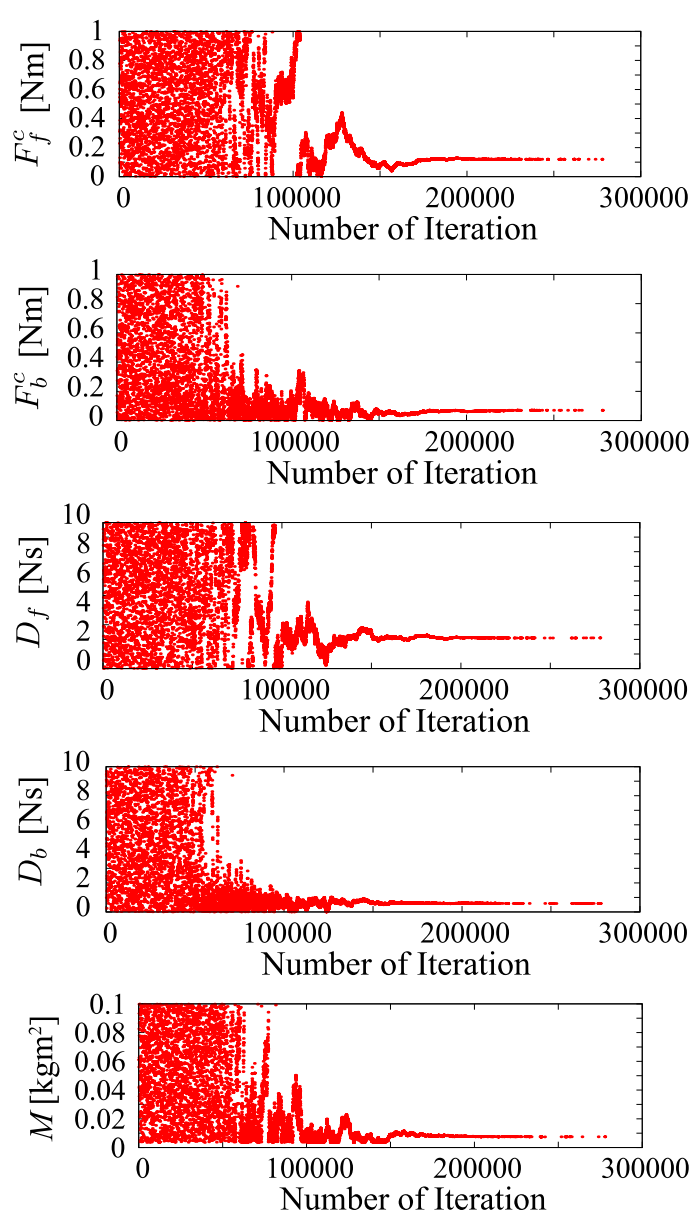

Fig. 12. Behavior of friction parameters in conventional research
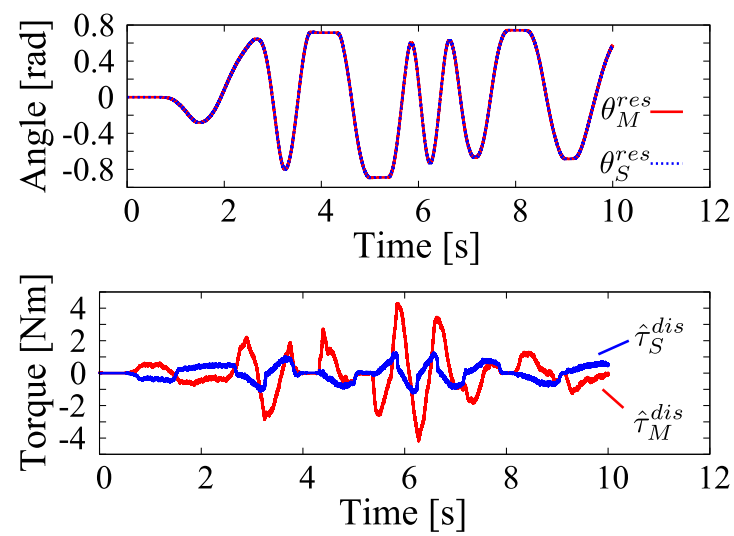

Fig. 13. Angle and torque responses of system with no compensation

$F_{b}^{c}, D_{f}, D_{b}$ and $M$ denote the friction parameters of conventional research. In addition, also the parameters determined by the simulated annealing don't necessarily correspond to the true optimized solution.

4.2 Experiments of Compensation In this part, the bilateral control with disturbance compensation is performed by RFOB based on determined parameters. In order to compensate for the varied self-inertia torque, the identified varied self-inertia torque is added to the original $J_{n}$. Therefore, in the experiment of compensation, $J_{n}$ is redefined 

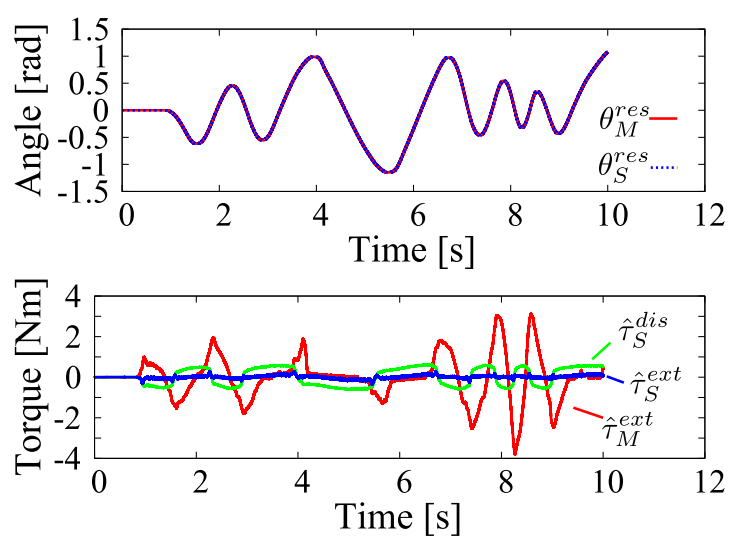

Fig. 14. Angle and torque responses of system with the compensation of the disturbance

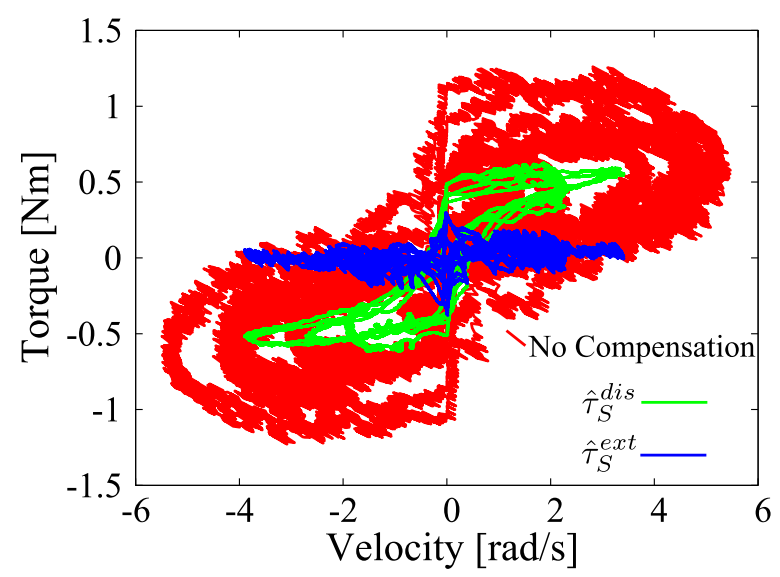

Fig. 15. Angle velocity-torque relation with compensation

as $0.00406 \mathrm{kgm}^{2}$. The cutoff frequency of RFOB is set to $300 \mathrm{rad} / \mathrm{s}$. The other experimental parameter values are same as Table 1.

The experimental results of system with no compensation and with compensation of the disturbance are shown in Figs. 13 and 14, respectively. The angle velocity-torque relation of each experimental result is shown in Fig. 15. In these figures, the angle responses represent trajectory of the experimental system. The torque responses represent torque information loaded to the system. $\tau^{d i s}$ denotes the output of DOB and represents the torque response that occurs in the actuator. However, in the experiment of compensation, a varied self-inertia torque is not included in the output because $J_{n}$ is redefined. $\tau^{e x t}$ denote the output of RFOB and represents the torque response that disturbance is compensated. In Fig. 15, No compensation, $\tau_{S}^{d i s}$, and $\tau_{S}^{e x t}$ denote $\tau_{S}^{d i s}$ in Fig. 13, $\tau_{S}^{d i s}$ in Fig. 14, and $\tau_{S}^{e x t}$ in Fig. 14, respectively. From comparing estimated value of RFOB with that of DOB in the slave system, it is found that the disturbance is well compensated. These results show the effectiveness of the proposed method. However, in spite of disturbance compensation, the large force response value of master system occurs. The reason is thought that the operational force has an effect on the force response ${ }^{(16)}$.

\section{Conclusions}

In this paper, the method of identification and compensation of the disturbance parameter values is proposed for improving the operationality of bilateral control. In this method, a constraint optimization problem was constructed for avoidance of an overcompensation to the actuator. The varied selfinertia torque, gravity and friction were considered as the disturbances of an actuator. Therefore, if large disturbances exist in an actuator such as geared motor, attainment of the realworld haptic system which can obtain high-accuracy tactile information is expected.

\section{Acknowledgment}

This research was partially supported by the Ministry of Education, Culture, Sports, Science and Technology, Grantin-Aid for Scientific Research for Young Scientists (A), 24686037, 2012.

\section{References}

( 1 ) K. Ohnishi: "Real World Haptics and Telehaptics for Medical Applications", 2010 IEEE International Symposium on Industrial Electronics (ISIE), pp.1619 (2010)

( 2 ) B. Hannaford and R. Anderson: "Experimental and Simulation Studies of Hard Contact in Force Reflecting Teleoperation", Proc. of the 1988 IEEE International Conference on Robotics and Automation, pp.584-589 (1988)

( 3 ) D.A. Lawrence: "Stability and Transparency in Bilateral Teleoperation", IEEE Trans. Robotics and Automation, Vol.9, No. 5, pp.624-637 (1993)

( 4 ) A. Sabanovic: "SMC Framework in Motion Control Systems", International Journal of Adaptive Control and Signal Processing, Vol.21, pp.731744 (2007)

( 5 ) M. Elitas, S. Khan, A.O. Nergiz, and A. Sabanovic: "Function Based Control for Bilateral Systems in Tele-Micromanipulation", AMC'08. 10th IEEE International Workshop on Advanced Motion Control, pp.544-549 (2008)

( 6 ) K. Ohnishi, M. Shibata, and T. Murakami: "Motion Control for Advanced Mechatronics", IEEE/ASME Transactions on Mechatronics, Vol.1, No.1, pp.56-67 (1996)

( 7 ) S. Katsura, Y. Matsumoto, and K. Ohnishi: "Modeling of Force Sensing and Validation of Disturbance Observer for Force Control", IEEE Trans. Industrial Electronics, Vol.54, No.1, pp.530-538 (2007)

( 8 ) T. Murakami, F. Yu, and K. Ohnishi: "Torque Sensorless Control in Multidegree-of-Freedom Manipulator", IEEE Trans. Industrial Electronics, Vol.40, No.2, pp.259-265 (1993)

( 9 ) S. Katsura, K. Irie, and K. Ohishi: "Wideband Force Control by PositionAcceleration Integrated Disturbance Observer", IEEE Trans. Industrial Electronics, Vol.55, No.4, pp.1699-1706 (2008)

(10) S. Katsura, Y. Matsumoto, and K. Ohnishi: "Analysis and Experimental Validation of Force Bandwidth for Force Control", IEEE Trans. Industrial Electronics, Vol.53, No.3, pp.922-928 (2006)

(11) S. Katsura and K. Ohishi: "Modal System Design of Multirobot Systems by Interaction Mode Control", IEEE Trans. Industrial Electronics, Vol.54, No.3, pp.1537-1546 (2007)

(12) Y. Yokokura, S. Katsura, and K. Ohishi: "Stability Analysis and Experimental Validation of a Motion-Copying System", IEEE Trans. Industrial Electronics, Vol.56, No.10, pp.3906-3913 (2009)

(13) S. Katsura and K. Ohnishi: "Quarry of Modal Information from Environment for Advanced Motion Control", IEEJ Trans. Industry Applications, Vol.126, No.4, pp.372-378 (2006)

(14) M.T. Eismann and R.C. Hardie: "Hyperspectral Resolution Enhancement Using High-Resolution Multispectral Imagery with Arbitrary Response Functions", IEEE Trans. Geoscience and Remote Sensing, Vol.43, No.3, pp.455465 (2005)

(15) T. Shimoichi and S. Katsura: "A Method of Friction Identification Based on Optimization Problem for Real-World Haptics", Japan Joint Automatic Control Conference (JACC 2011), No.2H404, pp.1430-1435 (2011)

(16) H. Tanaka and K. Ohnishi: "An Approach to Bilateral Control System for Improvement of Operationality", IEEJ Trans. Industry Applications, Vol.127, No.8, pp.851-857 (2007) 
Takuma Shimoichi (Student Member) received his B.E. degree in system design engineering and his M.E. degrees in integrated design engineering from Keio University, Yokohama, Japan, in 2010 and 2012, respectively. His research interests include real-world haptics, optimization and system identification.
Seiichiro Katsura (Senior Member) received his B.E. degree in sys-

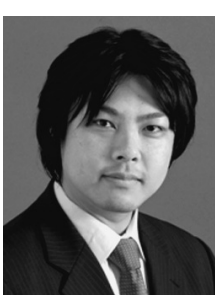
tem design engineering and his M.E. and Ph.D. degrees in integrated design engineering from Keio University, Yokohama, Japan, in 2001, 2002 and 2004, respectively. From 2003 to 2005, he was a Research Fellow of the Japan Society for the Promotion of Science. From 2005 to 2008, he worked at Nagaoka University of Technology, Nagaoka, Niigata, Japan. Since 2008, he has been at Keio University, Yokohama, Japan. His research interests include realworld haptics, human support space, systems energy conversion, and electromechanical integration systems. Prof. Katsura received the Best Paper Award from the Institute of Electrical Engineers of Japan (IEEJ) in 2003, the Dr. Yasujiro Niwa Outstanding Paper Award in 2004, The European Power Electronics and Drives-Power Electronics and Motion Control Conference, EPE-PEMC'08 Best Paper Award in 2008, and the IEEE Industrial Electronics Society Best Conference Paper Award in 2012. He is a Senior Member of IEEJ, as well as a Member of the IEEE, EPE, The Society of Instrument and Control Engineers (SICE), The Japan Society of Mechanical Engineers (JSME), The Japan Society for Precision Engineering (JSPE), Robotics Society of Japan (RSJ), The Institute of Electronics, Information and Communication Engineers (IEICE), and The Japan Society of Computer Aided Surgery (JSCAS). 